\title{
Adherence to the MIND dietary pattern and sleep quality, sleep related outcomes and mental health in male adults: a cross-sectional study
}

\author{
Hosein Rostami ${ }^{1}$, Karim Parastouei ${ }^{1 *}$, Mohammad Samadi ${ }^{2}$, Maryam Taghdir ${ }^{1}$ and Eslam Eskandari ${ }^{1}$
}

\begin{abstract}
Background: A large number of studies have investigated the association of the Mediterranean and DASH diets with psychological health as well as sleep related outcomes. However, only a few number of studies have examined the association of their newly proposed hybrid, Mediterranean-DASH Intervention for Neurodegenerative Delay (MIND) dietary pattern, with sleep quality and sleep related outcomes.

Methods: This cross-sectional study was conducted on 400 male health professions (mean age 38.67 years). Dietary information was collected using a validated 168-item semi-quantitative food frequency questionnaire. The MIND score was computed based on major dietary components emphasized or minimized in this pattern. The higher the MIND score of a subject, the greater his adherence to the MIND pattern. Mental health was evaluated using the 21-item depression, anxiety, and stress scale (DASS-21). Pittsburgh Sleep Quality Index (PSQI) was used to assess sleep quality. Sleep-related outcomes (day-time sleepiness and insomnia) were also evaluated using standard questionnaires
\end{abstract}

Results: No significant associations were found between adherence to the MIND diet score and odds of stress, anxiety and depression either in the crude or multivariable-adjusted models $(P>0.05)$. Nevertheless, participants with the greatest adherence to the MIND diet had lower odds of poor sleep quality (OR for T3 vs. T1: 0.56 ( $95 \%$ Cl: 0.34, 0.92), $P$-trend $=0.023$ ). The results remained significant after full adjustment for confounding variables (P-trend $=0.042$ ). Participants in the highest tertile of MIND diet had a $42 \%$ lower odds of daytime sleepiness in the crude and multivariable-adjusted model (P-trend $<0.05$ ). Although no significant association was observed between adherence to the MIND diet and severity of insomnia in the crude model (P-trend $=0.055)$, the multivariable-adjusted model showed that the highest adherence to the MIND diet was associated with lower odds of insomnia (OR for T3 vs. T1: 0.54 (95\% Cl: 0.31, 0.93), P-trend $=0.031$ ).

Conclusion: While no significant associations were found between adherence to the MIND diet and stress, anxiety and depression, greater adherence to the MIND diet were associated with lower odds of poor sleep quality and sleeprelated outcomes.

Keywords: MIND diet, Mental Health, Sleep quality, Insomnia, Daytime sleepiness, Cross-sectional

\footnotetext{
*Correspondence: parastouei@gmail.com

${ }^{1}$ Health Research Center, Life Style Institute, Baqiyatallah University

of Medical Sciences, Tehran, Iran

Full list of author information is available at the end of the article
} original author(s) and the source, provide a link to the Creative Commons licence, and indicate if changes were made. The images or other third party material in this article are included in the article's Creative Commons licence, unless indicated otherwise in a credit line to the material. If material is not included in the article's Creative Commons licence and your intended use is not permitted by statutory regulation or exceeds the permitted use, you will need to obtain permission directly from the copyright holder. To view a copy of this licence, visit http://creativecommons.org/licenses/by/4.0/. The Creative Commons Public Domain Dedication waiver (http://creativeco mmons.org/publicdomain/zero/1.0/) applies to the data made available in this article, unless otherwise stated in a credit line to the data. 


\section{Introduction}

The prevalence of mental disorders has been highlighted either in Iran [1] or worldwide [2] in the last decades. It is estimated that mental health-related disorders are accounting for approximately $7.4 \%$ of global burden diseases [2]. Research has indicated that mental health problems such as depression, anxiety, cognitive impairment, fatigue, and stress may be attributed to the poor quality and quantity of sleep and sleep disturbances [3-5]. In this regard, preventive strategies should be focused to tackle these mental health problems [6].

Lifestyle modifications including dietary components are considered as important primitive strategies in the prevention and treatment of psychological disorders [7]. In the recent past, the literature concerning the association of dietary components and mental health has gained more attention [8]. For instance, findings from a systematic review and meta-analysis of observational studies revealed that a healthy dietary pattern containing high intakes of fruits, vegetables, fish, and whole grains may be associated with a reduced depression risk, while no significant association was observed between the western dietary pattern and depression [9]. In addition, the results of a cross-sectional analysis in children and adolescents resulted in an increased risk of psychiatric distress and violent behaviors with higher intakes of junk foods [10]. In a cross-sectional study on 211 male adults, an association was found between poor dietary intakes and sleep disorders and insomnia [11].

Recently, a large number of studies have investigated the association of DASH and Mediterranean dietary patterns as healthy dietary patterns with psychological disorders. The results of a large prospective study indicated that consumption of Mediterranean dietary pattern was associated with lower risk of depressive symptoms in adults women [12]. On the other hand, higher adherence to DASH dietary pattern was inversely associated with daytime sleepiness score [13]. Recently, the combination of DASH and Mediterranean diet has been proposed as MIND diet (Mediterranean-DASH Diet Intervention for Neurodegenerative Delay) which emphasizes on dietary factors associated with psychological health [14]. Morris et al. found that higher MIND diet score is associated with slower decline in cognitive abilities and the MIND diet score is more predictive of cognitive decline than either of the DASH or Mediterranean diet score [14]. While only a limited number of observational studies have investigated the association of adherence to the MIND diet score and psychological disorders such as depression and anxiety, no study has been performed to investigate the association of adherence to the MIND diet with sleep quality and sleep-related outcomes. Thus, the present study is performed to examine the association between adherence to the MIND diet and psychological function in male Iranian adults.

\section{Methods}

This cross-sectional study was performed among Iranian male adults working in different healthcare centers affiliated to Baqiyatallah University of Medical Science across Tehran province from September 2020 to March 2021. Using a previous study performed by Rostami et al. [15] investigating the association of adherence to the DASH style diet and insomnia, the minimum required sample size was calculated as 323 , with alpha value and power set at 0.05 (2-sided) and $80 \%$, respectively. However, to increase the power of our study we recruited 400 individuals. A total of 400 male adults were randomly selected by using cluster sampling method among several healthcare centers. Male adults without any history of chronic diseases (colitis, diabetes, cardiovascular diseases, cancers, hepatic and renal failure, adrenal diseases, thyroid and parathyroid related disorders, anorexia nervosa or bulimia) were included. Those participants who received anti-diabetic, anti-inflammatory, anti-depressant or antiobesity drugs, vitamins and minerals supplements (for example: vitamin D or calcium and iron supplements) within the previous 6 months were also excluded. Written informed consent were taken from all the participants before running the study. The study was ethically approved by the Medical Ethics Committee of the Baqiyatallah University of Medical Science, Tehran, Iran (IR. BMSU.REC.1399.479).

\section{Dietary assessment}

Dietary data were collected using a semi-quantitative Willet-format food frequency questionnaire (FFQ) containing 168 food items with standard serving sizes [16]. The validity and reliability of this questionnaire in an Iranian population has been published previously [17]. Through an in person interview with expert nutritionists, participants were asked to report their consumption frequency of a given serving of each food item per day, week, month, or year. Afterwards, the obtained values of consumption were converted to the daily grams of intake for each food item using the Iranian manual for household measures [18]. The daily nutrient and energy intake of participants were calculated by entering the daily grams of intake of each food item into the Nutritionist-IV software (First Databank; Hearst, San Bruno, CA, USA). This nutrient database is based on the USDA food composition table modified for Iranian foods.

\section{Construction of MIND diet score}

The collected data by FFQ was used for constructing the MIND diet score. According to the method of MIND 
diet, 15 dietary components consist of 10 brain-healthy food groups (green leafy vegetables, other vegetables, nuts, berries, beans, whole grains, fish, poultry, olive oil, and wine) and 5 unhealthy food groups ( red meats and red meat products, butter and stick margarine, cheese, pastries, fast fried foods and sweets) are included in this dietary score. In the current study, wine was not included in the MIND diet due to the lack of information in the FFQs. To build the MIND diet score, subjects were initially classified into tertile categories based on the intakes of these food components. Subjects in the lowest tertile of brain-healthy food groups (green leafy vegetables, other vegetables, nuts, berries, beans, whole grains, fish and poultry intake) were scored 0 , those in the middle tertile were given the score of 0.5 and those in the highest tertile were given the score of 1 . In case of brain-unhealthy food groups (red meats and red meat products, butter and margarine, cheese, pastries, fast fried foods and sweets), the exact opposite of this scoring protocol was applied. The scores of all 14 food components were then added up to construct an overall MIND score ranging theoretically from 0 to 14 for each subject. The higher the MIND diet score of a subject, the greater his adherence to the MIND pattern.

\section{Demographic and anthropometric assessments}

A standard questionnaire was used to obtain general demographic information including age, smoking status (yes/ no), marital status (single/ married), smoking status (yes/ no), education (elementary or lower/ high school/ college and university) by a face to face interview. Physical activity was obtained using International Physical Activity Questionnaire (IPAQ)-short form and expressed as metabolic equivalent (MET)-min/day [19]. The validity and reliability of the IPAQ has been investigated in Iranian population [20]. Height was measured to the nearest $0.1 \mathrm{~cm}$ using a wall-fixed measuring tape. Body weight was measured while participants were in light clothes and without shoes. Body mass index (BMI) was calculated by dividing weight $(\mathrm{kg})$ to height $\left(\mathrm{m}^{2}\right)$.

\section{Depression Anxiety Stress Scales (DASS)}

Depression Anxiety and Stress Scale (DASS-21) was used to screen depression, anxiety and stress. DASS21 is a valid and reliable questionnaire to measure status of negative well beings for general population [21]. The questionnaire contains 21 items with 3 subscales. Each subscale includes 7 questions in which each item rates on a 4-point Likert scale $0-3$ measuring severity of depression, anxiety and stress. Higher scores shows severe negative emotion. The total score of each sub-class should be doubled since DASS-21 is the short version of DASS-42 [21]. All three main domains were devided into two categories (no anxiety (score $\leq 7)$ or some degree of anxiety $(>7)$; no depression ( score $\leq 9$ ) or some degree of depression (>9); no stress ( score $\leq 14$ ) or some degree of stress $(>14)$. The validated DASS-21 questionnaire in an Iranian population was used in the current study [22].

\section{Sleep quality assessment}

Pittsburgh Sleep Quality Index (PSQI) was used as a reliable and valid tool to assess sleep quality. This tool consists 19 questions with seven components scores: sleep quality, sleep latency, sleep duration, habitual sleep efficiency, sleep disturbances, use of sleeping medication, and daytime dysfunction. Each component score is rated from 0 to $3(0$, not in the past month; 1 , less than once per week; 2 , once or twice per week; and 3 , three or more times per week). The total score ranges from 0 to 21, which higher scores indicating poorer sleep quality. Total scores greater than 5 indicated poor sleep quality while scores $\leq 5$ were indicative of normal sleep quality. The validated version of this tool in an Iranian population done by farrahi et al. with acceptable validity and reliability was used in the current study [23].

\section{Assessment of daytime sleepiness}

The persian validated form of Epworth Sleepiness Scale (ESS) was used for the assessment of daytime sleepiness [24]. The questionnaire includes 8 questions investigating sleepiness in eight daily situations. Each question is rated from 0 to 3 giving a total score of $0-24$. The higher scores indicating the more excessive daytime sleepiness. The severity of sleepiness was considered as normal $($ score $\leq 10)$ and mild to moderate sleepiness $($ score $>10)$. The validity and reliability of ESS questionnaire in an Iranian population has been published elsewhere [25].

\section{Assessment of insomnia}

The Insomnia Severity Index (ISI) questionnaire was used to assess sleep insomnia. The ISI questionnaire has seven questions with a score range of $0-4$ for each item which is stratified into four categories as follows: 0 (none), 1 (mild), 2 (moderate), 3 (severe) and 4 (very Severe). The total score ranges between 0 to 28 points and the ISI score $>7$, is considered to have insomnia. The validated ISI questionnaire in an Iranian population was used in the current study [26].

\section{Statistical analysis}

Baseline characteristics of participants across tertiles of the MIND diet score were expressed as means \pm SDs for continuous variables and percentages for categorical variables. Subjects were classified into three categories based on tertiles of MIND diet score. The Chi-square test was used for comparison of categorical variables and 
the one-way analysis of variance (ANOVA) was used for comparison of continuous variables among tertiles of MIND score. Dietary intakes of study.

Participants across tertiles of the MIND score were compared using ANCOVA, adjusted for total energy intake, except for dietary energy intake. Stress, anxiety, depression, poor sleep quality, insomnia and daytime sleepiness scores were divided into two categories (no/ minimal degree and some degree of disorder) regarding to scores. We used binary logistic regressions to estimate ORs and 95\% CIs for the presence of mentioned outcomes across tertiles of the MIND score in crude and multivariable-adjusted models. Age, BMI, and total energy intake were controlled for in the first model. Additional adjustments were controlled for physical activity and smoking (yes/ no). Finally, further adjustment were applied for marital status (single/ married) and educational levels (elementary or lower/ high school/ college and university) in the third model. A P-value $<0.05$ was considered as statistically significant. All statistical analyses were done using SPSS (SPSS Inc., version 22).

\section{Results}

The general characteristics of 400 included participants are presented in Table 1. Mean age and BMI of whole population was $38.67 \pm 5.26$ year and $25.28 \pm 3.22 \mathrm{~kg} /$ $\mathrm{m}^{2}$. Individuals with lower adherence to MIND diet were more physically active (Table 1 ). No significant differences were observed between tertiles of MIND score for age, BMI, marital status, education levels and smoking status (Table 1).

Dietary intakes of study participants across tertiles of MIND score are provided in Table 2. Participants in the third tertile had higher intakes of beans, berries, dietary fiber, vegetables, white meat, whole grains as well as total calorie compared with those in the first tertile $(P<0.05)$. In addition, dietary intakes of red meats and total fat were significantly different across tertiles of MIND diet $(P<0.05)$.

Crude and multivariable-adjusted ORs (95\% CIs) for stress, anxiety and depression across tertiles of MIND diet score are indicated in Table 3. No significant associations were observed between adherence to the MIND diet and stress in crude model (OR for T3 vs. T1: 0.73 (95\% CI: 0.44, 1.19), P-trend $=0.206$ ) or multivariable adjusted model (OR for T3 vs. T1: 0.75 (95\% CI: $0.44,1.26)$, P-trend $=0.280$ ). The crude or multivariable adjusted model between the MIND diet and anxiety revealed no significant associations, (OR for T3 vs. T1: 0.82 (95\% CI: 0.50, 1.34), P-trend $=0.425)$ and (OR for T3 vs. T1: 0.81 (95\% CI: 0.48, 1.36), P-trend $=429)$, respectively. In addition, there was no significant

Table 1 Baseline characteristics of participants according to tertiles of MIND diet score $(n=400)$

\begin{tabular}{|c|c|c|c|c|}
\hline \multirow[t]{2}{*}{ Variables } & \multicolumn{3}{|c|}{ Tertiles of MIND diet score } & \multirow[t]{2}{*}{$P$-value ${ }^{b}$} \\
\hline & $<4.25(n=130)$ & $4.25-6.15(n=130)$ & $>6.16-11.5(n=140)$ & \\
\hline Age $\left(\right.$ years) ${ }^{a}$ & $38.66 \pm 5.12$ & $38.60 \pm 5.32$ & $38.72 \pm 5.38$ & 0.983 \\
\hline Body weight & $78.73 \pm 9.09$ & $78.85 \pm 8.34$ & $78.10 \pm 10.76$ & 0.779 \\
\hline BMI $\left(\mathrm{kg} / \mathrm{m}^{2}\right)$ & $25.05 \pm 2.79$ & $25.49 \pm 3.12$ & $25.29 \pm 3.68$ & 0.543 \\
\hline Physical-activity (MET-min/day) & $117.82 \pm 37.65$ & $104.93 \pm 47.06$ & $112.14 \pm 43.00$ & 0.52 \\
\hline Work shift (hours/day) & $7.31 \pm 1.44$ & $7.06 \pm 1.27$ & $7.38 \pm 1.25$ & 0.113 \\
\hline \multicolumn{5}{|l|}{ Marital status } \\
\hline Single & $28(21.5 \%)$ & $23(17.7 \%)$ & $21(15 \%)$ & \multirow[t]{2}{*}{0.381} \\
\hline Married & $102(78.5 \%)$ & $107(82.3 \%)$ & $119(85.0 \%)$ & \\
\hline \multicolumn{5}{|l|}{ Smoking status } \\
\hline Yes & $5(3.8 \%)$ & $6(4.6 \%)$ & $6(4.3 \%)$ & \multirow[t]{2}{*}{1.000} \\
\hline No & 125 (96.2\%) & 124 (95.4\%) & $134(95.7 \%)$ & \\
\hline \multicolumn{5}{|l|}{ Education } \\
\hline Elementary or lower & $32(24.6 \%)$ & $38(29.2 \%)$ & $40(28.6 \%)$ & \multirow[t]{3}{*}{0.818} \\
\hline High school & $67(51.5 \%)$ & $68(52.3 \%)$ & $70(50.0 \%)$ & \\
\hline College and university & $31(23.8 \%)$ & $24(18.5 \%)$ & $30(21.4 \%)$ & \\
\hline \multicolumn{4}{|l|}{ Home ownership } & \multirow[t]{3}{*}{0.140} \\
\hline Owner & 119 (91.5\%) & 109 (83.8\%) & 125 (89.3\%) & \\
\hline Tenant & 11 (8.5\%) & $21(16.2 \%)$ & 15 (10.7\%) & \\
\hline
\end{tabular}

${ }^{a}$ Data are mean \pm standard deviation (SD)

${ }^{b}$ Obtained from ANOVA or chi-square test, where appropriate 
Table 2 Dietary intakes of study participants across tertiles of MIND diet score ${ }^{a}(n=400)$

\begin{tabular}{lccc}
\hline Variables & \multicolumn{2}{l}{ Tertiles of MIND diet score } & \\
\cline { 2 - 3 } & T1 (<4.25) & T2 (4.25-6.15) & T3 (>6.16-11.5) \\
\hline Food groups (gr/day) & & & \\
$\quad$ Vegetables & $227.97 \pm 12.35$ & $251.10 \pm 12.29$ & $296.23 \pm 12.30$ \\
Beans & $28.18 \pm 6.08$ & $39.12 \pm 6.05$ & $45.51 \pm 6.05$ \\
Berries & $19.39 \pm 5.69$ & $25.71 \pm 5.66$ & $51.10 \pm 5.66$ \\
Nuts & $28.18 \pm 6.08$ & $39.12 \pm 6.05$ & $45.51 \pm 6.05$ \\
Whole grains & $9.31 \pm 1.69$ & $12.53 \pm 1.68$ & $17.21 \pm 1.68$ \\
Red meat and red meat products & $62.64 \pm 7.23$ & $61.82 \pm 7.20$ & $89.68 \pm 7.20$ \\
White meat & $18.82 \pm 3.15$ & $24.96 \pm 3.13$ & $36.32 \pm 3.13$ \\
sweets & $165.93 \pm 10.74$ & $163.35 \pm 10.70$ & $188.44 \pm 10.58$ \\
Nutrients & & & 0.136 \\
Total energy (kcal/day) & $1964.93 \pm 67.38$ & $2009.11 \pm 67.38$ & 0.006 \\
Carbohydrate (gr/day) & $373.75 \pm 30.30$ & $379.28 \pm 30.15$ & $2509.39 \pm 64.93$ \\
Protein (gr/day) & $92.74 \pm 10.13$ & $95.87 \pm 10.08$ & $412.10 \pm 30.16$ \\
Fat (gr/day) & $90.82 \pm 3.22$ & $79.82 \pm 3.21$ & $99.38 \pm 10.08$ \\
Fiber (gr/day) & $31.68 \pm 2.35$ & $36.07 \pm 2.34$ & $89.66 \pm 3.21$ \\
Folate ( $\mu$ g/day) & $477.92 \pm 38.41$ & $526.44 \pm 38.22$ & 0.001 \\
Vitamin B6 (mg/day) & $2.05 \pm 0.205$ & $2.29 \pm 0.204$ & 0.202 \\
Vitamin B12 ( $\mu$ g/day) & $10.98 \pm 6.75$ & $20.71 \pm 6.72$ & $602.83 \pm 38.24$ \\
Magnesium (mg/day) & $377.28 \pm 14.91$ & $388.15 \pm 14.84$ & $2.5 \pm 0.204$ \\
\hline
\end{tabular}

${ }^{a}$ Data are Mean \pm standard error (SE)

* All values were adjusted for energy intake, except for dietary energy intake, using ANCOVA

Table 3 Crude and multivariable-adjusted odds ratio (95\% Cl) of the associations between MIND-style diet and stress, anxiety and depression

\begin{tabular}{cllll}
\hline & T1 $(\boldsymbol{n = 1 3 0 )}$ & T2 $(\boldsymbol{n = 1 3 0 )}$ & T3 $(\boldsymbol{n = 1 4 0})$ & P-trend \\
\hline Stress & & & & \\
Crude & $\mathbf{1 . 0 0}$ & $0.90(0.54-1.50)$ & $0.73(0.44-1.19)$ & 0.206 \\
Model I & $\mathbf{1 . 0 0}$ & $0.90(0.54-1.50)$ & $0.75(0.44-1.25)$ & 0.270 \\
Model II & $\mathbf{1 . 0 0}$ & $0.96(0.58-1.61)$ & $0.78(0.46-1.30)$ & 0.346 \\
Model III & $\mathbf{1 . 0 0}$ & $0.94(0.56-1.57)$ & $0.75(0.44-1.26)$ & 0.280 \\
Anxiety & $\mathbf{1 . 0 0}$ & & & \\
Crude & $\mathbf{1 . 0 0}$ & $1.03(0.62-1.72)$ & $0.82(0.50-1.34)$ & 0.425 \\
Model I & $\mathbf{1 . 0 0}$ & $1.03(0.62-1.72)$ & $0.80(0.48-1.35)$ & 0.419 \\
Model II & $\mathbf{1 . 0 0}$ & $1.01(0.60-1.70)$ & $0.80(0.47-1.34)$ & 0.400 \\
Model III & $\mathbf{1 . 0 0}$ & $1.02(0.61-1.72)$ & $0.81(0.48-1.36)$ & 0.429 \\
Depression & $\mathbf{1 . 0 0}$ & & & \\
Crude & $\mathbf{1 . 0 0}$ & $1.10(0.66-1.85)$ & $0.81(0.49-1.34)$ & 0.412 \\
Model I & $\mathbf{1 . 0 0}$ & $1.11(0.66-1.87)$ & $0.78(0.46-1.32)$ & 0.370 \\
Model II & $\mathbf{1 . 0 0}$ & $1.11(0.66-1.89)$ & $0.78(0.46-1.33)$ & 0.374 \\
Model III & $\mathbf{1 . 0 0}$ & $1.09(0.64-1.85)$ & $0.76(0.45-1.29)$ & 0.315 \\
\hline
\end{tabular}

Data are OR $(95 \% \mathrm{Cl})$

Model I: adjusted for age, BMI, energy intake Model II: Model I + smoking, physical activity Model III: Model II + marital status, education association between MIND diet and depression in crude (OR for T3 vs. T1: 0.81 (95\% CI: 0.49, 1.34), P-trend $=0.412$ ) and fully-adjusted model (OR for T3 vs. T1: 0.76 (95\% CI: 0.44, 1.49), P-trend $=0.315)$.

Table 4 indicates the crude and multivariableadjusted ORs for sleep quality, daytime sleepiness and insomnia across tertiles of MIND diet. Using a crude model, the odds of poor sleep quality reduced significantly with increasing adherence to the MIND diet (OR for T3 vs. T1: 0.56 (95\% CI: 0.34, 0.92), $\mathrm{P}$-trend $=0.023)$. The results remained significant after full adjustment for confounding variables (OR for T3 vs. T1: 0.58 (95\% CI: 0.34, 0.98), P-trend $=0.042$ ). Participants in the highest tertile of MIND diet had a $42 \%$ lower odds of daytime sleepiness in the crude (95\% CI: 0.34, 0.98; P-trend $=0.031$ ) and multivariable adjusted model (95\% CI: 0.35, 0.98; P-trend $=0.044)$. Although no significant association was observed between adherence to the MIND diet and severity of insomnia in the crude model (OR for T3 vs. T1: 0.60 (95\% CI: 0.36, 1.01), P-trend $=0.055)$, the multivariable adjusted model showed that highest adherence to the MIND diet was associated with lower odds of insomnia (OR for T3 vs. T1: 0.54 (95\% CI: 0.31, 0.93), P-trend =0.031). 
Table 4 Crude and multivariable-adjusted odds ratio (95\% Cl) of the associations between MIND-style diet and sleep quality, daytime sleepiness and insomnia

\begin{tabular}{|c|c|c|c|c|}
\hline & $\mathrm{T} 1(n=130)$ & T2 $(n=130)$ & T3 $(n=140)$ & P-trend \\
\hline \multicolumn{5}{|l|}{ Poor sleep quality } \\
\hline Crude & 1.00 & $0.78(0.47-1.31)$ & $0.56(0.34-0.92)^{*}$ & 0.023 \\
\hline Model I & 1.00 & $0.77(0.46-1.30)$ & $0.56(0.33-0.95)^{*}$ & 0.032 \\
\hline Model II & 1.00 & $0.78(0.46-1.31)$ & $0.56(0.33-0.95)^{*}$ & 0.033 \\
\hline Model III & 1.00 & $0.79(0.47-1.34)$ & $0.58(0.34-0.98)^{*}$ & 0.042 \\
\hline Insomnia & 1.00 & & & \\
\hline Crude & 1.00 & $0.90(0.55-1.49)$ & $0.60(0.37-0.98)^{*}$ & 0.039 \\
\hline Model I & 1.00 & $0.91(0.55-1.50)$ & $0.56(0.33-0.93)^{*}$ & 0.027 \\
\hline Model II & 1.00 & $0.88(0.53-1.47)$ & $0.55(0.33-0.92)^{*}$ & 0.024 \\
\hline Model III & 1.00 & $0.88(0.53-1.46)$ & $0.54(0.32-0.91)^{*}$ & 0.022 \\
\hline Daytime sleepiness & 1.00 & & & \\
\hline Crude & 1.00 & $0.63(0.38-1.05)$ & $0.58(0.35-0.94)^{*}$ & 0.031 \\
\hline Model I & 1.00 & $0.64(0.39-1.06)$ & $0.60(0.36-1.01)$ & 0.055 \\
\hline Model II & 1.00 & $0.63(0.38-1.05)$ & $0.60(0.36-1.00)$ & 0.053 \\
\hline Model III & 1.00 & $0.62(0.37-1.03)$ & $0.58(0.35-0.98)^{*}$ & 0.044 \\
\hline
\end{tabular}

Data are OR $(95 \% \mathrm{Cl})$

Model I: adjusted for age, BMI, energy intake

Model II: Model I + smoking, physical activity

Model III: Model II + marital status, education

${ }^{*} P$-value $<0.05$

\section{Discussion}

To the best of our knowledge, the current study is the first report investigating the association between adherence to the MIND dietary pattern and sleep quality as well as sleep outcomes including insomnia and daytime sleepiness among adults. As stated in the methods section, our study was performed among Iranian male adults working in different healthcare centers affiliated to Baqiyatallah University of Medical Science, which is a military dependent organization in Iran. Thus, the majority of personnel were men. The association of dietary patterns and sleep quality and sleep related outcomes are of great importance in those who are working in military service or military related organizations due to the fact that they are at higher risk of sleep related disorders $[27,28]$. Thus, we tried to address this associations in this group of individuals in the current study. Our results indicated that greater adherence to the MIND dietary pattern was associated with better sleep quality. In addition, an inverse association was found between adherence to the MIND diet and severity of insomnia and daytime sleepiness.

Dietary modifications may have a practical role in not only improving sleep quality but also in preventing sleep disorders. The findings of a cross-sectional study on 410 female students in Iran found a significant association between short sleep duration and low quality of diet [29]. Another study on 896 American women revealed no significant relationship between short sleep duration and diet quality, while significant associations were observed between long sleep duration and lower diet quality, specially lower fruit intakes and higher consumption of empty calories [30].

As reported in our results, higher intake of vegetables, beans, berries and whole grains were observed in subjects with higher adherence to the MIND diet and higher adherence to the MIND diet was associated with better sleep quality and less sleep disorders including insomnia and daytime sleepiness. In line with our findings, results of previous studies have investigated that higher intake of specific dietary components (fibers, minerals and vitamins) are associated with better sleep duration and quality and less sleep disorders [31, 32]. The results of a cross-sectional study on 1936 Italian individuals revealed that adherence to a low inflammatory index diet (such as DASH or MIND diet) was associated with improved sleep quality [33]. Pahlavani et al. investigated the correlation between adherence to the DASH dietary pattern and daytime sleepiness score in 535 adolescent girls in Iran; the results of the their study shed light on the inverse association between adherence to DASH diet and daytime sleepiness score [13]. Another similar study on 488 adolescent girls in Iran revealed that high adherence to the DASH-style 
diet was associated with a lower odds of insomnia in comparison to subjects with the lowest adherence [15].

Evidence has indicated that quality of sleep, sleep duration and sleep disturbance are associated with systemic inflammation [34]. The mechanisms that might explain the associations between sleep disturbance and inflammation are not fully explored. Sleep affects two primary effector systems, the sympathetic nervous system (SNS) and the hypothalamus-pituitary-adrenal (HPA) axis, which both together shift the basal gene expression profile toward increased pro-inflammatory profile $[35,36]$. Activation of $\beta$-adrenergic signaling stimulates NF- $\kappa B$ pathway, inflammatory gene expression, release of proinflammatory cytokines, and markers of systemic inflammation [35]. Since normal nocturnal sleep is associated with a reduction in sympathetic outflow [37], the sympathetic effector pathway activation might be considered as one of the potential mechanisms to explain the associations between sleep disturbance, short sleep duration, and increased systemtic inflammation. On the other hand, MIND dietary pattern as a hybrid of Mediterranean and DASH dietary patterns contains a variety of anti-inflmmatory components including: omega-3 fatty acids in fish and walnuts, folate in grean leafy vegetables, vitamin $\mathrm{E}$ in nuts and olive oil, and flavonoids in berries which may exert greater anti-inflammatory effects with regard to the mentioned dietary patterns independently. Therefore, the anti-inflammatory effects of MIND dietary pattern may justify the favorable effects on sleep quality and daytime sleepiness and insomnia. In addition, evidence has indicated that sleep disturbance may accumulate free radicals in the cerebral tissues which subsequently leads to cerebral oxidative stress [38]. It has also proven that MIND dietary pattern has antioxidant effects which support its preventive effects on cognitive impairment and sleep disorders [39]. Given the role of oxidative stress in the pathogenesis of psychological and sleep-related disorders [40, 41], the antioxidant and anti-inflammatory properties of MIND diet might protect brain health. MIND diet characterized by a limited amounts of unhealthy foods including red meats, butter, fast foods, pastries and sweets which are harmful for brain health, while it is rich in fruits and vegetables as rich sources of vitamins, minerals, flavonoids and antioxidants [14].

As reported in our results, no significant associations were found between adherence to the MIND dietary pattern and depression, anxiety and stress in the current study. Although a considerable number of studies have performed to investigate the association between adherence to the DASH and Mediterranean dietary patterns and depression, anxiety and stress, only a limited number of studies have investigated the association between MIND dietary pattern and mental health. For instance, the results of a cross-sectional study on 3176 adults found an inverse association between greater adherence to the MIND diet and odds of depression and psychological distress, while similar to our findings, no significant association was reported between adherence to the MIND diet and odds of anxiety [42]. Faghih et al. performed a cross-sectional study on 240 university students and concluded that subjects in the highest tertile of DASH score had lower means score for depression, anxiety and stress compared to those in the lowest tertile [43]. The results of another study on 580 adolescent girls revealed significant inverse association between greater adherence to the DASH diet and lower odds of depression, however, found no significant relationship between a DASH-style diet and aggression [6]. Furthermore, findings from Sadeghi et al. study on 3172 adults aged $18-55$ years found that participants with the highest adherence to the Mediterranean diet had lower odds for anxiety, depression, and psychological distress compred to subjects in the lowest adherence [44].

This study has several strengths. To our best knowledge, this is the first study investigating the linkage between MIND diet score and sleep quality and sleeprelated outcomes. It is also among the first studies which have investigated the association between MIND dietary pattern and depression, anxiety and stress. The statistical analysis were controlled for several potential confounders. In addition, dietary intakes were obtained using a validated and reliable FFQ. On the other hand, the current study has some limitations that should be considered while interpreting the findings. The current study can not prove the causality and the temporality of relations due to the nature of study design (cross-sectional study). Our findings can be generalizable only to a small group of populations, males health professions, and might not be generalizable to females or other populations. Moreover, study outcomes were assessed using self-reported questionnaires and are susceptible to recall bias and misreporting.

In conclusion, no significant associations were found between adherence to the MIND diet and odds of stress, anxiety and depression in this cross-sectional study on male adults. Nevertheless, our findings indicate that greater adherence to the MIND diet were associated with lower odds of poor sleep quality, daytime sleepiness and insomnia. Given the limitations of the current study, further investigations of sufficient methodological quality are needed to confirm these findings.

Acknowledgements

Not applicable. 


\section{Authors' contributions}

H. R: Study design, writing first draft, and data analysis, K. P: Study design, editing, and final checking, M. S: Outcomes assessing and manuscript editing, M. T: Outcomes assessing and manuscript editing, E. E: Data collection and proofreading. The authors readand approved the final manuscript.

\section{Funding}

None.

\section{Availability of data and materials}

The data used and analyzed during the current study are available from the corresponding author on reasonable request.

\section{Declarations}

\section{Ethics approval and consent to participate}

Current study was approved by the ethics committee of Baqiyatallah University of Medical Sciences with the approval code of IR.BMSU.REC.1399.479. All methods were conducted in accordance with the relevant guidelines and regulations. In addition, all participants signed a written informed consent form to participate in the study before enrollment.

\section{Consent for publication}

Not applicable.

\section{Competing interests}

There are no conflicts of interest.

\section{Author details}

${ }^{1}$ Health Research Center, Life Style Institute, Baqiyatallah University of Medical Sciences, Tehran, Iran. ${ }^{2}$ Present Address: Exercise Physiology Research Center, Life Style Institute, Baqiyatallah University of Medical Sciences, Tehran, Iran.

Received: 24 September 2021 Accepted: 28 February 2022

Published online: 05 March 2022

\section{References:}

1. Mohammadi M-R, Davidian H, Noorbala AA, Malekafzali H, Naghavi HR, Pouretemad HR, et al. An epidemiological survey of psychiatric disorders in Iran. Clin Pract Epidemiol Ment Health. 2005;1(1):1-8.

2. Whiteford HA, Degenhardt L, Rehm J, Baxter AJ, Ferrari AJ, Erskine HE, et al. Global burden of disease attributable to mental and substance use disorders: findings from the Global Burden of Disease Study 2010. The lancet. 2013;382(9904):1575-86.

3. Staner L. Sleep and anxiety disorders. Dialogues Clin Neurosci. 2003;5(3):249.

4. Tsuno N, Besset A, Ritchie K. Sleep and depression. J Clin Psychiatry. 2005:66(10):1254-69.

5. Reid KJ, Martinovich Z, Finkel S, Statsinger J, Golden R, Harter K, et al. Sleep: a marker of physical and mental health in the elderly. Am J Geriatr Psychiatry. 2006;14(10):860-6.

6. Khayyatzadeh SS, Mehramiz M, Mirmousavi SJ, Mazidi M, Ziaee A, KazemiBajestani SMR, et al. Adherence to a Dash-style diet in relation to depression and aggression in adolescent girls. Psychiatry Res. 2018;259:104-9.

7. O'neil A, Quirk SE, Housden S, Brennan SL, Williams LJ, Pasco JA, et al. Relationship between diet and mental health in children and adolescents: $a$ systematic review. Am J Public Health. 2014;104(10):e31-42.

8. Haghighatdoost F, Azadbakht L, Keshteli AH, Feinle-Bisset C, Daghaghzadeh $\mathrm{H}$, Afshar $\mathrm{H}$, et al. Glycemic index, glycemic load, and common psychological disorders. Am J Clin Nutr. 2016;103(1):201-9.

9. Lai JS, Hiles S, Bisquera A, Hure AJ, McEvoy M, Attia J. A systematic review and meta-analysis of dietary patterns and depression in communitydwelling adults. Am J Clin Nutr. 2014;99(1):181-97.

10. Zahedi H, Kelishadi R, Heshmat R, Motlagh ME, Ranjbar SH, Ardalan G, et al. Association between junk food consumption and mental health in a national sample of Iranian children and adolescents: the CASPIAN-IV study. Nutrition. 2014;30(11-12):1391-7.
11. Tan X, Alén M, Cheng SM, Mikkola TM, Tenhunen J, Lyytikäinen A, et al. Associations of disordered sleep with body fat distribution, physical activity and diet among overweight middle-aged men. J Sleep Res. 2015;24(4):414-24.

12. Rienks J, Dobson A, Mishra G. Mediterranean dietary pattern and prevalence and incidence of depressive symptoms in mid-aged women: results from a large community-based prospective study. Eur J Clin Nutr. 2013;67(1):75-82.

13. Pahlavani N, Khayyatzadeh SS, Banazadeh V, Bagherniya M, Tayefi M, Eslami S, et al. Adherence to a dietary approach to stop hypertension (DASH)-style in relation to daytime sleepiness. Nature and Science of Sleep. 2020;12:325.

14. Morris MC, Tangney CC, Wang Y, Sacks FM, Barnes LL, Bennett DA, et al. MIND diet slows cognitive decline with aging. Alzheimers Dement. 2015;11(9):1015-22.

15. Rostami H, Khayyatzadeh SS, Tavakoli H, Bagherniya M, Mirmousavi SJ, Farahmand SK, et al. The relationship between adherence to a Dietary Approach to Stop Hypertension (DASH) dietary pattern and insomnia. BMC Psychiatry. 2019;19(1):1-7.

16. Willett W. Nutritional epidemiology: Oxford university press; 2012

17. Esfahani FH, Asghari G, Mirmiran P, Azizi F. Reproducibility and relative validity of food group intake in a food frequency questionnaire developed for the Tehran Lipid and Glucose Study. J Epidemiol. 2010;20(2):150-8.

18. Ghaffarpour M, Houshiar-Rad A, Kianfar H. The manual for household measures, cooking yields factors and edible portion of foods. Tehran: Nashre Olume Keshavarzy 1999;7 213:42 58.

19. Craig CL, Marshall AL, Sjöström M, Bauman AE, Booth ML, Ainsworth BE, et al. International physical activity questionnaire: 12-country reliability and validity. Med Sci Sports Exerc. 2003;35(8):1381-95.

20. Moghaddam MB, Aghdam FB, Jafarabadi MA, Allahverdipour $\mathrm{H}$, Nikookheslat SD, Safarpour S. The Iranian Version of International Physical Activity Questionnaire (IPAQ) in Iran: content and construct validity, factor structure, internal consistency and stability. World Appl Sci J. 2012;18(8):1073-80.

21. Henry JD, Crawford JR. The short-form version of the Depression Anxiety Stress Scales (DASS-21): Construct validity and normative data in a large non-clinical sample. Br J Clin Psychol. 2005;44(2):227-39.

22. Sahebi A, Asghari MJ, Salari RS. Validation of depression anxiety and stress scale (DASS-21) for an Iranian population. 2005.

23. Moghaddam JF, Nakhaee N, Sheibani V, Garrusi B, Amirkafi A. Reliability and validity of the Persian version of the Pittsburgh Sleep Quality Index (PSQI-P). Sleep and Breathing. 2012;16(1):79-82.

24. Johns MW. A new method for measuring daytime sleepiness: the Epworth sleepiness scale. Sleep. 1991;14(6):540-5.

25. Haghighi KS, Montazeri A, Mehrizi AK, Aminian O, Golkhandan AR, Saraei $M$, et al. The Epworth Sleepiness Scale: translation and validation study of the Iranian version. Sleep and Breathing. 2013;17(1):419-26.

26. Yazdi Z, Sadeghniiat-Haghighi K, Zohal MA, Elmizadeh K. Validity and reliability of the Iranian version of the insomnia severity index. The Malaysian journal of medical sciences: MJMS. 2012;19(4):31.

27. Sayari R, Sanayinasab H, Ebadi A, Rahmati Najarkolaei F, Chavoshi F. Sleep disorders in military nurses. Journal of Police Medicine. 2014;3(3):149-58.

28. Brown CA, Berry R, Schmidt A. Sleep and military members: emerging issues and nonpharmacological intervention. Sleep Disord. 2013;2013:160374.

29. Haghighatdoost F, Karimi G, Esmaillzadeh A, Azadbakht L. Sleep deprivation is associated with lower diet quality indices and higher rate of general and central obesity among young female students in Iran. Nutrition. 2012:28(11-12):1146-50.

30. Xiao RS, Simas TAM, Pagoto SL, Person SD, Rosal MC, Waring ME. Sleep duration and diet quality among women within 5 years of childbirth in the United States: a cross-sectional study. Matern Child Health J. 2016;20(9):1869-77.

31. Grandner MA, Jackson N, Gerstner JR, Knutson KL. Sleep symptoms associated with intake of specific dietary nutrients. J Sleep Res. 2014;23(1):22-34

32. Peuhkuri K, Sihvola N, Korpela R. Diet promotes sleep duration and quality. Nutr Res. 2012;32(5):309-19. 
33. Godos J, Ferri R, Caraci F, Cosentino Fl, Castellano S, Shivappa N, et al. Dietary inflammatory index and sleep quality in Southern Italian adults. Nutrients. 2019;11(6):1324.

34. Irwin MR, Olmstead R, Carroll JE. Sleep disturbance, sleep duration, and inflammation: a systematic review and meta-analysis of cohort studies and experimental sleep deprivation. Biol Psychiat. 2016;80(1):40-52.

35. Irwin MR, Cole SW. Reciprocal regulation of the neural and innate immune systems. Nat Rev Immunol. 2011;11(9):625-32.

36. Slavich GM, Irwin MR. From stress to inflammation and major depressive disorder: a social signal transduction theory of depression. Psychol Bull. 2014;140(3):774.

37. Irwin M, Thompson J, Miller C, Gillin JC, Ziegler M. Effects of sleep and sleep deprivation on catecholamine and interleukin-2 levels in humans: clinical implications. J Clin Endocrinol Metab. 1999;84(6):1979-85.

38. Gulec M, Ozkol H, Selvi Y, Tuluce Y, Aydin A, Besiroglu L, et al. Oxidative stress in patients with primary insomnia. Prog Neuropsychopharmacol Biol Psychiatry. 2012;37(2):247-51.

39. Hosking DE, Eramudugolla R, Cherbuin N, Anstey KJ. MIND not Mediterranean diet related to 12-year incidence of cognitive impairment in an Australian longitudinal cohort study. Alzheimers Dement. 2019;15(4):581-9.

40. Salim S. Oxidative stress and psychological disorders. Curr Neuropharmacol. 2014;12(2):140-7.

41. Hachul de Campos H, Brandao L, D'Almeida V, Grego B, Bittencourt L, Tufik $\mathrm{S}$, et al. Sleep disturbances, oxidative stress and cardiovascular risk parameters in postmenopausal women complaining of insomnia. Climacteric. 2006;9(4):312-9.

42. Salari-Moghaddam A, Keshteli AH, Mousavi SM, Afshar H, Esmaillzadeh A, Adibi P. Adherence to the MIND diet and prevalence of psychological disorders in adults. J Affect Disord. 2019;256:96-102.

43. Faghih S, Babajafari S, Mirzaei A, Akhlaghi M. Adherence to the dietary approaches to stop hypertension (DASH) dietary pattern and mental health in Iranian university students. Eur J Nutr. 2020;59(3):1001-11.

44. Sadeghi O, Keshteli AH, Afshar H, Esmaillzadeh A, Adibi P. Adherence to Mediterranean dietary pattern is inversely associated with depression, anxiety and psychological distress. Nutr Neurosci. 2021;24(4):248-59.

\section{Publisher's Note}

Springer Nature remains neutral with regard to jurisdictional claims in published maps and institutional affiliations.

Ready to submit your research? Choose BMC and benefit from:

- fast, convenient online submission

- thorough peer review by experienced researchers in your field

- rapid publication on acceptance

- support for research data, including large and complex data types

- gold Open Access which fosters wider collaboration and increased citations

- maximum visibility for your research: over 100M website views per year

At BMC, research is always in progress.

Learn more biomedcentral.com/submissions 